\title{
Simultaneous UV- and ion processing of astrophysically relevant ices The case of $\mathrm{CH}_{3} \mathrm{OH}: \mathrm{N}_{2}$ solid mixtures
}

\author{
F. Islam, G. A. Baratta, and M. E. Palumbo
}

\begin{abstract}
INAF, Osservatorio Astrofisico di Catania, via Santa Sofia 78, 95123 Catania, Italy e-mail: mepalumbo@oact.inaf.it
\end{abstract}

Received 3 June 2013 / Accepted 20 November 2013

\section{ABSTRACT}

\begin{abstract}
Context. Interstellar ices are known to be simultaneously processed by both cosmic-ray bombardment and UV photolysis. Our knowledge of the effects of energetic processing on relevant icy samples is mainly based on laboratory investigations. In the past 35 years many experiments have been performed to study these effects separately but, to the best of our knowledge, never simultaneously. Aims. The aim of this work is to study the effects of simultaneous processing of ices by both cosmic rays and UV photons to investigate to what extent the combined effect of ion bombardment and UV photolysis influences the chemical pathways.

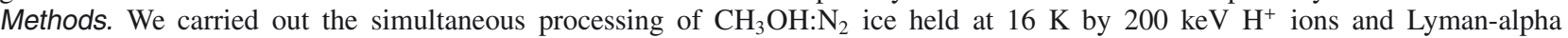
$10.2 \mathrm{eV}$ UV photons. The samples were analyzed by in situ transmission infrared spectroscopy. The un-combined processes of UV irradiation and bombardment by $\mathrm{H}^{+}$ions of $\mathrm{CH}_{3} \mathrm{OH}: \mathrm{N}_{2}$ ice were also studied. This mixture was chosen because the effects of ion bombardment and UV photolysis on methanol and nitrogen have been extensively studied in previous investigations. This mixture enables one to investigate whether simultaneous processing (a) influences the destruction of original species; (b) influences the formation of new species; or (c) causes synergistic effects since Lyman-alpha photons have a very low efficiency in breaking the dinitrogen bond because $\mathrm{N}_{2}$ is almost transparent at Lyman-alpha wavelengths.

Results. After processing a $\mathrm{CH}_{3} \mathrm{OH}: \mathrm{N}_{2}$ sample, the intensity of the methanol bands was observed to decrease at the same rate in all cases. After ion bombardment, species such as $\mathrm{CO}_{2}, \mathrm{CO}, \mathrm{H}_{2} \mathrm{CO}, \mathrm{CH}_{4}, \mathrm{~N}_{2} \mathrm{O}, \mathrm{HNCO}$, and $\mathrm{OCN}^{-}$are formed in the ice mixture. After UV photolysis, species such as $\mathrm{CO}_{2}, \mathrm{CO}, \mathrm{H}_{2} \mathrm{CO}$, and $\mathrm{CH}_{4}$ are formed, but no N-bearing species are detected. Spectra of ices processed by both UV photons and ions were compared with spectra of ices bombarded only by ions. We find that there are no differences in the band area and profile of N-bearing species for the two types of experiment at the same ion fluence; therefore, the addition of UV irradiation to ion bombardment does not affect the abundance of N-bearing species. The initial formation rate of $\mathrm{CH}_{4}$, within the experimental uncertainties, is the same in all cases studied, while the saturation value of $\mathrm{CH}_{4}$ is higher for UV photolysis than for ion bombardment when they act separately. In the case of simultaneous processing, when the dose $(\mathrm{eV} / 16 \mathrm{u})$ given by UV photons is similar to the dose given during ion bombardment, the saturation value of $\mathrm{CH}_{4}$ reaches a value intermediate between the value obtained after UV photolysis and ion bombardment separately.

Conclusions. Our results confirm that when UV photolysis and ion bombardment act separately, their effects are very similar from a qualitative point of view, while significant quantitative difference may exist. In the case of simultaneous processing we did not detect any synergistic effect, but in some instances the behavior of newly formed species (such as $\mathrm{CH}_{4}$ ) can significantly depend on the $\mathrm{UV} /$ ions dose ratio.
\end{abstract}

Key words. astrochemistry - molecular processes - methods: laboratory - techniques: spectroscopic - ISM: molecules

\section{Introduction}

The key to interstellar chemistry lies in the formation of molecules on dust grain surfaces. These dust grains are thought to be mainly silicate or carbonaceous in nature. In dense regions, such as in the interior of dense molecular clouds, dust grains provide shielding from the external galactic UV radiation field, allowing the formation of icy mantles surrounding the dust grains. The interior of molecular clouds is penetrated by cosmic rays, which are ubiquitous throughout the interstellar medium. Cosmic rays may be protons, alpha particles, nuclei of heavy atoms, electrons, and positrons (Swordy 2001). The interaction of interstellar material and cosmic rays in turn produce a number of secondary particles, such as low-energy cosmic rays, electrons, and UV photons. Thus, interstellar ices are processed simultaneously by mostly internal but also by attenuated external UV radiation as well as by fast-moving ions from the galactic cosmic radiation field. Both processes induce chemical and structural changes within the ice mantles of interstellar dust grains (e.g., Grim et al. 1989; Jenniskens et al. 1993; Palumbo et al. 1998; Hudson et al. 2008; Palumbo et al. 2010).

The distinct processes of ion bombardment and UV irradiation on solids of astrophysical interest are well characterized (e.g., Hagen et al. 1979; Brown et al. 1982; Moore et al. 1983; Strazzulla et al. 1983a; Allamandola et al. 1988; Baratta et al. 1991; Westley et al. 1995a; Gerakines et al. 1996; Strazzulla et al. 2001; Moore et al. 2001; Leto \& Baratta 2003; Öberg et al. 2009; Modica \& Palumbo 2010; Garozzo et al. 2011). Some investigations on the comparison between the two processes have also been carried out. Experimental results have shown that from a qualitative point of view, ion bombardment and UV photolysis generate similar changes in interstellar ice analogs. However, quantitative differences between the two processes have been observed. Cottin et al. (2001) examined organic residues produced from $\mathrm{H}_{2} \mathrm{O}: \mathrm{CO}: \mathrm{CH}_{3} \mathrm{OH}: \mathrm{NH}_{3}$ ices after UV photolysis or proton bombardment to find that hexamethylenetetramine was present in the residue after both processes. Gerakines et al. (2000) investigated the formation of 
carbonic acid $\left(\mathrm{H}_{2} \mathrm{CO}_{3}\right)$ from $\mathrm{H}_{2} \mathrm{O}: \mathrm{CO}_{2}$ ice mixtures and found that $\mathrm{H}_{2} \mathrm{CO}_{3}$ is readily formed by proton bombardment. However, in the case of UV photolysis, the penetration of UV photons into the ice limits the production of $\mathrm{H}_{2} \mathrm{CO}_{3}$. Gerakines \& Moore (2001) investigated the formation of carbon suboxide $\left(\mathrm{C}_{3} \mathrm{O}_{2}\right)$ in ices analogous to cometary nuclei and interstellar regions to give formation and destruction rates for $\mathrm{C}_{3} \mathrm{O}_{2}, \mathrm{CO}$ and $\mathrm{CO}_{2}$ for both proton bombardment and UV irradiation. They found that Lyman-alpha photons are 3.5 times more efficient than $800 \mathrm{keV}$ protons in synthesizing $\mathrm{CO}_{2}$ on pristine $\mathrm{CO}$ per unit incident energy. Differently to Gerakines \& Moore (2001), Loeffler et al. (2005) analyzed their data as a function of absorbed energy and found that the production rates (i.e., molecules formed per unit of absorbed energy) of $\mathrm{CO}_{2}$, formed after processing of $\mathrm{CO}$ ice, by both $200 \mathrm{keV}$ protons and Lyman-alpha photons are similar, but at saturation (i.e., when the exponential trend approaches the asymptotic value) the concentration of $\mathrm{CO}_{2}$ is three times higher for ion bombardment.

In studies of $\mathrm{N}_{2}$ ices, Hudson \& Moore (2002) discovered that while the $\mathrm{N}_{3}$ (azide) radical was readily formed after bombardment with $0.8 \mathrm{MeV}$ protons, photolysis by far-UV did not result in the detection of $\mathrm{N}_{3}$. However, studies by $\mathrm{Wu}$ et al. (2012) have shown that UV photolysis of thick (about $160 \mu \mathrm{m}$ ) solid $\mathrm{N}_{2}$ samples does indeed produce $\mathrm{N}_{3}$, thus it is most likely that some $\mathrm{N}_{3}$ was produced in the experiments of Hudson \& Moore, albeit in very small quantities below their detection limit. Furthermore, Kobayashi et al. (2008) irradiated gas mixtures of carbon monoxide, nitrogen, and water using UV light and particle beams of hydrogen (protons), helium, carbon, neon, and argon ions to investigate the production of amino acids. They found that when $\mathrm{N}_{2}$ molecules were used as the nitrogen source, amino acids were not detected after photolysis with UV light, but were detected after processing with the particle beams. Kobayashi et al. ascribed this effect to the inability of UV light to dissociate either $\mathrm{CO}$ or $\mathrm{N}_{2}$ molecules. From the results reported by Wu et al. (2012), assuming the density of $\mathrm{N}_{2}$ to be about $0.83 \mathrm{~g} \mathrm{~cm}^{-3}$ (Roux et al. 1980), it is possible to estimate that after UV photolysis, at saturation (i.e., when the exponential trend approaches the asymptotic value) the ratio between the column densities of $\mathrm{N}_{3}$ and initial $\mathrm{N}_{2}$ is about $10^{-6}$. Sicilia et al. (2012) reported the results of $200 \mathrm{keV}$ proton bombardment of solid $\mathrm{N}_{2}$. From their results, assuming that the band strength value for the $1657 \mathrm{~cm}^{-1}$ feature of $\mathrm{N}_{3}$ is $4 \times 10^{-17} \mathrm{~cm} / \mathrm{molecule}$ (Jamieson \& Kaiser 2007), it is possible to estimate that after ion bombardment with $200 \mathrm{keV}$ protons at saturation the ratio between the column densities of $\mathrm{N}_{3}$ and initial $\mathrm{N}_{2}$ is about $10^{-4}$. These experimental results show that given the same initial column density of $\mathrm{N}_{2}$, the column density of $\mathrm{N}_{3}$ after ion bombardment is two orders of magnitude higher than after UV photolysis.

Therefore, although ion bombardment and UV photolysis both induce similar chemical changes to interstellar ice, there are marked differences in the yields of the two processes, and as yet, it is unknown whether the two processes can affect each other.

To the best of our knowledge, only one combined experiment has been conducted with both ion bombardment and UV photolysis of interstellar-relevant ices. Baratta et al. (2002) investigated the destruction of $\mathrm{CH}_{4}$ and $\mathrm{CH}_{3} \mathrm{OH}$ by $30 \mathrm{keV} \mathrm{He}^{+}$ions and $10.2 \mathrm{eV}$ Lyman-alpha photons. The effects of the two processes were found to be comparable at low doses $(\leq 20 \mathrm{eV} / 16 \mathrm{u})$, such that the same number of $\mathrm{CH}_{4}$ and $\mathrm{CH}_{3} \mathrm{OH}$ molecules are destroyed at the same doses (see their Figs. 4 and 7). However, at high doses $(>20 \mathrm{eV} / 16 \mathrm{u})$, UV photolysis is less efficient in the destruction of $\mathrm{CH}_{4}$ and $\mathrm{CH}_{3} \mathrm{OH}$, whereas ion bombardment continues to induce chemical changes. This is because a residue is formed after continuous exposure to ion bombardment and UV irradiation (Moore et al. 1983; Strazzulla et al. 1983a; Strazzulla \& Johnson 1991; Jenniskens et al. 1993; Ferini et al. 2004). The formation of the residue changes the optical properties of the pristine material that becomes progressively optically thick to UV radiation as processing continues. This causes the absorption coefficient to increase, therefore most UV photons are absorbed by the uppermost layers of the sample and do not penetrate the bulk of the ice, whereas the release of energy from ions into the ice remains unaffected. When a sample of $\mathrm{CH}_{4}$ ice was first irradiated with UV up to a dose of $35 \mathrm{eV} / 16 \mathrm{u}$ and was then bombarded with $30 \mathrm{keV} \mathrm{He} \mathrm{He}^{+}$ions (from $37 \mathrm{eV} / 16 \mathrm{u}$ to $89 \mathrm{eV} / 16 \mathrm{u}$ ) in a combined experiment, Baratta et al. (2002) found that the rate of $\mathrm{CH}_{4}$ destruction by ions between doses of $40 \mathrm{eV} / 16 \mathrm{u}$ to $60 \mathrm{eV} / 16 \mathrm{u}$ increased in comparison with an experiment where the ice was bombarded by ions alone (see their Fig. 5). $\mathrm{CH}_{4}$ molecules were found to be more efficiently destroyed by ion bombardment in an ice sample that had previously undergone UV photolysis. Therefore, it is possible that UV photolysis can enhance the process of ion bombardment.

The concept of synergy between different energetic processes in space has been considered in previous work. Westley et al. (1995a and 1995b) irradiated water-ice with Lyman-alpha photons to investigate photodesorption. They speculated that sputtering of ices by ions may be enhanced due to precursor radicals produced by UV photolysis, which may explain the difference between observations of $\mathrm{OH}$ near Tethys, a satellite of Saturn (Shemansky et al. 1993), and sputtering calculations (Johnson et al. 1993). In addition, they considered the possibility that an impinging cosmic ray could cause a transient temperature increase to enhance the sublimation of photolyzed ice to augment photodesorption rates.

As yet, it is unknown to what extent the combined effect of ion bombardment and UV photolysis influences the chemical pathways in icy mixtures. In this paper, we aim to investigate the effect of the occurrence of these two processes in tandem and separately on interstellar ices. To simplify this study we have considered the case of a $\mathrm{CH}_{3} \mathrm{OH}: \mathrm{N}_{2}$ mixture. This mixture was chosen because the effects of ion bombardment and UV photolysis on methanol have been extensively studied in previous investigations (e.g., Gerakines et al. 1996; Baratta et al. 2002) and because Lyman-alpha photons have a very low efficiency in breaking the dinitrogen bond (Hudson \& Moore 2002; Moore \& Hudson 2003; Wu et al. 2012) because $\mathrm{N}_{2}$ is almost transparent at Lyman-alpha wavelengths. Based on the experimental results reported by Moore \& Hudson (2003) and Wu et al. (2012), we expect that the number of $\mathrm{N}$-bearing species formed by UV photolysis is on the order of $10^{-4}$ with respect to pristine species. This amount is too low to be detected in the experimental conditions adopted in this work. On the other hand, fast ions can efficiently break $\mathrm{N}_{2}$ molecules, giving rise to a significant number of N-bearing species. At this point, $\mathrm{N}$-bearing radicals and molecules could be excited and/or broken by Lyman-alpha photons, giving rise to detectable additional (i.e., synergistic) effects. For clarity, throughout the manuscript we use the term ion bombardment to indicate ion processing, and UV photolysis or UV irradiation to indicate UV processing. The term processing is used to mean either process.

\section{Experimental method}

The experimental apparatus has been described in detail elsewhere (Strazzulla et al. 2001; Baratta et al. 2002; Palumbo et al. 2004). Experiments were carried out in a stainless steel 


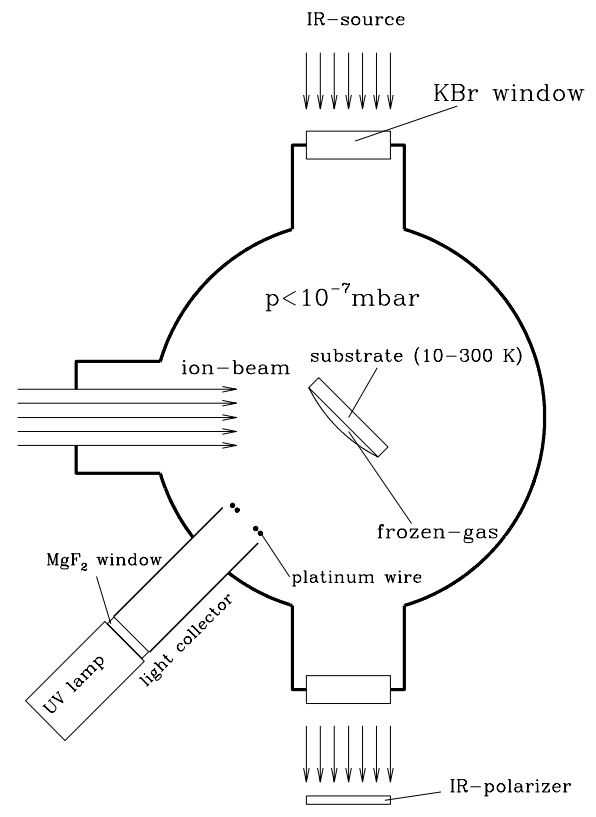

IR-detector

Fig. 1. Schematic view of the experimental apparatus used for in situ IR spectroscopy of frozen gases after ion bombardment and UV photolysis.

vacuum chamber shown in Fig. 1 at a base pressure of about $10^{-7}$ mbar. Transmission spectra were taken using a Bruker Equinox 55 FTIR spectrometer at a resolution of $1 \mathrm{~cm}^{-1}$ in the range $4400-400 \mathrm{~cm}^{-1}(2.27-25 \mu \mathrm{m})$. A silicon substrate of thickness $500 \mu \mathrm{m}$ was cooled to $16 \mathrm{~K}$ by a cold-finger in thermal contact with a closed-cycle helium cryostat. Gases were admitted into the vacuum chamber through a needle valve, resulting in a background deposition of ice onto the silicon crystal. This method has the advantage that the film deposited has an uniform thickness but has the disadvantage that gas phase molecules can freeze out onto all cold surfaces inside the chamber. To avoid any deposition on the backside of the substrate, this is protected by a $2 \mathrm{~cm}$ long copper tube aligned with the IR beam and with the central hole with a diameter of about $4.5 \mathrm{~mm}$. The thickness of the deposited ice was determined from the interference fringe curve of a reflected He-Ne laser beam following Baratta \& Palumbo (1998), Fulvio et al. (2009), and Modica \& Palumbo (2010).

Bombardment by $200 \mathrm{keV}$ protons was performed using an electrostatic $200 \mathrm{kV}$ ion implanter (Danfysik) with magnetic mass separation. The ion beams were scanned electrostatically to ensure a uniform fluence over the sample. The ion beam and the infrared beam were mutually perpendicular and formed an angle of 45 degrees with the substrate plane, thus before, during, and after bombardment, spectra could be obtained in situ without tilting the sample. The ion beam produced a spot on the target larger than the area probed by the IR beam. The current density of the ion beam must be sufficiently low to avoid macroscopic heating of the sample and typically ranged from $100 \mathrm{nA} \mathrm{cm}^{-2}$ to a few $\mu \mathrm{A} \mathrm{cm}^{-2}$. The ions passed through the sample without stopping, as the penetration depth of impinging ions is known to be much deeper than the sample thickness from SRIM calculations (Ziegler et al. 2008). Typically, the thickness of our samples was about $250 \mathrm{~nm}$ and the penetration depth of $200 \mathrm{keV} \mathrm{H}^{+}$ in a $\mathrm{CH}_{3} \mathrm{OH}: \mathrm{N}_{2}$ mixture was about $2 \mu \mathrm{m}$.
Photolysis of the sample by UV photons was carried out by means of a microwave-discharge hydrogen lamp interfaced with the vacuum chamber through a $\mathrm{MgF}_{2}$ window. The discharge was produced by a continuous Opthos microwave generator operating at $2.45 \mathrm{GHz}$. The lamp was operated with a gas mixture of $\mathrm{H}_{2}(30 \%)$ and $\mathrm{Ar}(70 \%)$ and a gas pressure $P=0.5$ mbar. The lamp used in this work as well as the operating conditions were the same as used by Baratta et al. (2002) and Loeffler et al. (2005). An aluminium light collector was placed at the end of the lamp to increase the number of UV photons that reached the sample. We considered that the discharge lamps are not monochromatic and that the spectrum of the emitted radiation depends on experimental conditions, such as pressure in the discharge tube and gas mixture ratio. For example, the operating conditions adopted by Westley et al. (1995b, see their Fig. 1) produced UV photons mainly at Lyman-alpha wavelength. The operating conditions of the UV lamp adopted in this work were very similar to those used by Westley et al. (1995a,b), and we assumed that most of the photons were emitted at $121.6 \mathrm{~nm}$ (10.2 eV, Lyman-alpha). We developed a monitoring system of the UV flux to obtain a reliable measure of the total number of UV photons that reach the sample during photolysis (see Baratta et al. 2002). This system is described in detail in the online appendix. The flux of the lamp can be adjusted in the range $10^{12}-5 \times 10^{14} \mathrm{~cm}^{-2} \mathrm{~s}^{-1}$. The typical fluence of UV photons used is on the order of $10^{18} \mathrm{~cm}^{-2}$.

In this study $\mathrm{CH}_{3} \mathrm{OH}$ and $\mathrm{N}_{2}$ were coadsorbed on a silicon substrate held at $16 \mathrm{~K}$ in a sandwich between layers of Ar to protect the sample from contamination by water and other background gases in the chamber. On the basis of different calibration experiments we have shown that contamination species are $\mathrm{H}_{2} \mathrm{O}$ and $\mathrm{CO}_{2}$, which deposit on top of the sample during processing (Loeffler et al. 2005). After further processing, CO can be formed from $\mathrm{CO}_{2}$. Because of the Ar layer, these additional deposits do not affect the chemical changes induced by processing, which take place in the bulk of the sample. As shown by Loeffler et al. (2005), the deposition of background gases is significantly larger during UV photolysis than ion bombardment. In particular, after a photon fluence equal to $2.5 \times 10^{18}$ photons $\mathrm{cm}^{-2}$, the $\mathrm{CO}_{2}$ column density can be as high as $2 \times 10^{16}$ molecules $\mathrm{cm}^{-2}$ (Loeffler et al. 2005). As a consequence of this phenomenon, in this study we did not consider the trend of $\mathrm{CO}$ and $\mathrm{CO}_{2}$ column density during processing.

The IR spectra may be taken by selecting the parallel ( $\mathrm{P}$ polarization) or perpendicular ( $\mathrm{S}$ polarization) component of the electric vector with respect to the plane of incidence using a rotatable polarizer placed in front of the detector. For each experiment, background spectra in $\mathrm{P}$ and $\mathrm{S}$ polarization were acquired before ice deposition and subtracted from all spectra with the same polarization (Baratta \& Palumbo 1998; Baratta et al. 2000). $\mathrm{P}$ polarization spectra are presented here because the signal-tonoise ratio is higher than for $\mathrm{S}$ polarization.

\section{Results}

We deposited about $250 \mathrm{~nm}$ thick $\mathrm{CH}_{3} \mathrm{OH}: \mathrm{N}_{2}$ ice sandwiched between two Ar layers on a silicon substrate held at $16 \mathrm{~K}$. The samples were processed by $200 \mathrm{keV} \mathrm{H}^{+}$and $10.2 \mathrm{eV}$ Lymanalpha photons separately and in tandem. For the simultaneous processing, two different experiments were performed with a different ratio between UV and ion fluence. A list of the experiments is given in Table 1.

Figure 2 shows the spectra, in optical depth scale, relative to the $\mathrm{CH}_{3} \mathrm{OH}: \mathrm{N}_{2}$ mixture as deposited (panel a), after ion 
Table 1. Details of experiments.

\begin{tabular}{|c|c|c|c|c|}
\hline Processing & $\begin{array}{l}\text { Max UV fluence } \\
\text { (photons } \mathrm{cm}^{-2} \text { ) }\end{array}$ & $\begin{array}{l}\text { Max UV dose }{ }^{a} \\
(\mathrm{eV} / 16 \mathrm{u})\end{array}$ & $\begin{array}{l}\text { Max ion fluence } \\
\quad \text { (ions } \mathrm{cm}^{-2} \text { ) }\end{array}$ & $\begin{array}{c}\text { Max ion dose }{ }^{a} \\
(\mathrm{eV} / 16 \mathrm{u})\end{array}$ \\
\hline Ion bombardment & - & - & $1.36 \times 10^{15}$ & 36 \\
\hline UV photolysis & $3.25 \times 10^{18}$ & 55 & - & - \\
\hline Simultaneous & $1.76 \times 10^{18}$ & 34 & $1.29 \times 10^{14}$ & 3 \\
\hline Simultaneous & $1.76 \times 10^{18}$ & 33 & $8.54 \times 10^{14}$ & 23 \\
\hline
\end{tabular}

Notes. ${ }^{(a)}$ Here the dose is the energy released to the target molecules in $\mathrm{eV}$ per small molecule (16u), where $\mathrm{u}$ is the unified atomic mass unit (see text).



Fig. 2. IR transmittance spectra in optical-depth scale (P polarization) of $\mathrm{CH}_{3} \mathrm{OH}: \mathrm{N}_{2}$ ice (about $250 \mathrm{~nm}$ thick) deposited at $16 \mathrm{~K}$ as deposited; after bombardment with $3.91 \times 10^{14} 200 \mathrm{keV} \mathrm{H}^{+} \mathrm{cm}^{-2}$ (dose $=10 \mathrm{eV} / 16 \mathrm{u}$ ); after photolysis with $2.42 \times 10^{18}$ photons $\mathrm{cm}^{-2}$ (dose $\left.=41 \mathrm{eV} / 16 \mathrm{u}\right)$; after simultaneous processing with $1.29 \times 10^{14} 200 \mathrm{keV} \mathrm{H}^{+} \mathrm{cm}^{-2}$ and $1.76 \times 10^{18}$ photons $\mathrm{cm}^{-2}$ (total dose $\left.=37 \mathrm{eV} / 16 \mathrm{u}\right)$.

bombardment (panel b), after UV photolysis (panel c), and after simultaneous processing (panel d). Changes in the intensity of the methanol bands at $1039 \mathrm{~cm}^{-1}$ (C-O stretch), $1129 \mathrm{~cm}^{-1}$ (- $\mathrm{CH}_{3}$ rock), $1460 \mathrm{~cm}^{-1}(\mathrm{C}-\mathrm{H}$ deformation and $\mathrm{O}-\mathrm{H}$ bend $)$, $2830 \mathrm{~cm}^{-1}$ (symmetric C-H stretch), $2961 \mathrm{~cm}^{-1}$ (asymmetric $\mathrm{C}-\mathrm{H}$ stretch), and $3260 \mathrm{~cm}^{-1}$ (O-H stretch) indicate that destruction of methanol occurs after processing.

To compare the effects that are induced by fast ions and UV photons, the irradiation dose was derived (energy released onto the target molecules, i.e., $\mathrm{eV}$ molecule ${ }^{-1}$ ). As suggested by Strazzulla \& Johnson (1991), the dose given in units of eV per small molecule (16u) is a convenient way to characterize chemical changes and to compare the results obtained after processing of different samples. The energy released into the sample by impinging ions or photons (dose) is then given in $\mathrm{eV} / 16 \mathrm{u}$, where $\mathrm{u}$ is the unified atomic mass unit defined as $1 / 12$ of the mass of an isolated atom of carbon-12. For the ion bombardment,

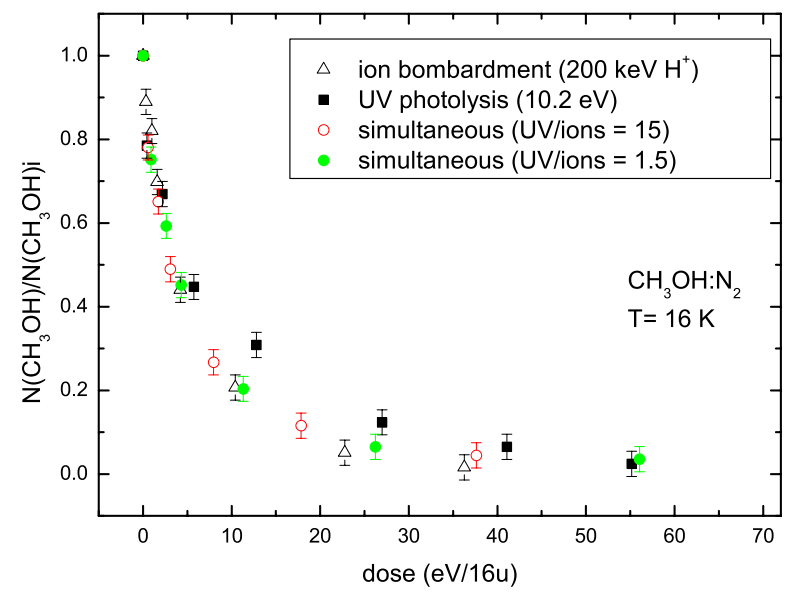

Fig. 3. Normalized methanol column density as a function of dose for the UV photolysis, ion bombardment, and simultaneous processing.

the dose $(\mathrm{eV} / 16 \mathrm{u})$ was obtained from the ion fluence values (ions $\mathrm{cm}^{-2}$ ) measured during the experiment and the stopping power $S\left(\mathrm{eV} \mathrm{cm}^{2}\right.$ molecule $\left.{ }^{-1}\right)$ calculated using the SRIM code (Ziegler et al. 2008). For the UV photolysis, the dose (eV/16u) was obtained from the UV fluence (photons $\mathrm{cm}^{-2}$ ) measured during the experiment and the absorption cross-section of Lymanalpha photons in $\mathrm{CH}_{3} \mathrm{OH}\left(\sigma_{a}=6.37 \times 10^{-18} \mathrm{~cm}^{2}\right)$ given by Baratta et al. (2002) assuming that the absorption coefficient of Lyman-alpha photons in $\mathrm{N}_{2}$ is negligible. Two experiments with simultaneous processing were performed. In the first experiment, the dose given by UV photons was on average 15 times higher than the dose given by $200 \mathrm{keV}$ protons. In the second experiment the dose ratio was about 1.5 .

After processing the pristine $\mathrm{CH}_{3} \mathrm{OH}: \mathrm{N}_{2}$ ice, the IR spectra showed a reduction in methanol abundance. Figure 3 shows the column density of methanol normalized to the initial methanol column density as a function of dose for the UV photolysis, ion bombardment, and simultaneous processing. The column density of methanol was derived from the band at about $1039 \mathrm{~cm}^{-1}$ after the transmittance spectrum was converted to optical depth scale and using the band strength value $A=1.3 \times 10^{-17} \mathrm{~cm} \mathrm{molecule}{ }^{-1}$ (Palumbo et al. 1999). As discussed by Baratta et al. (2002), at low doses the trend of methanol column density is very similar in all cases, while at high doses the destruction of methanol is slightly slower in the case of UV photolysis. This effect can be ascribed to the change of the optical properties of the sample during processing. The optical constants of the sample change in all cases due to energetic processing. It is well known that $\mathrm{C}$-bearing species evolve toward a polymer-like material and eventually to a refractory residue (Moore et al. 1983; Strazzulla et al. 1983a; Strazzulla \& Johnson 1991; Jenniskens et al. 1993; Ferini et al. 2004). The 
F. Islam et al.: Simultaneous UV- and ion processing of ice mixtures

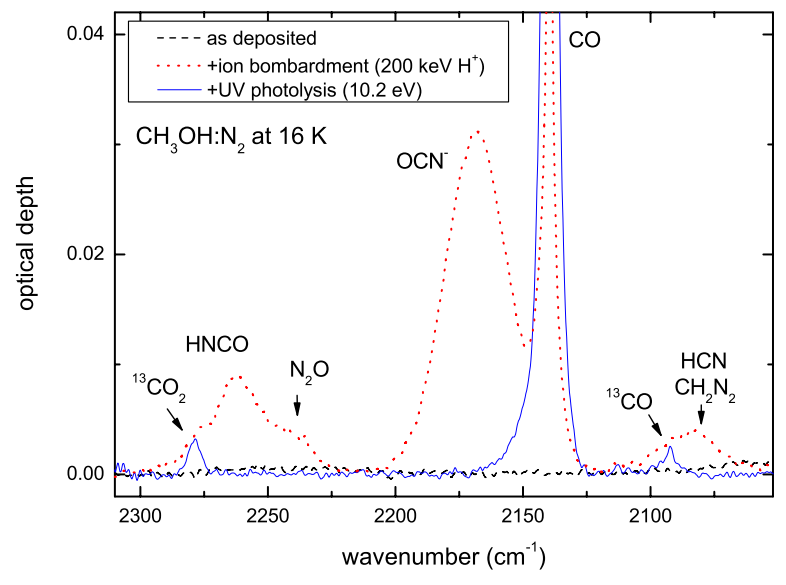

Fig. 4. IR transmittance spectra in optical-depth scale ( $\mathrm{P}$ polarization) of $\mathrm{CH}_{3} \mathrm{OH}: \mathrm{N}_{2}$ ice (about $250 \mathrm{~nm}$ thick) deposited at $16 \mathrm{~K}$ before processing (dashed line), after bombardment with $1.36 \times 10^{15}$ $200 \mathrm{keV} \mathrm{H}^{+} \mathrm{cm}^{-2}$ (dose $=36 \mathrm{eV} / 16 \mathrm{u}$; dotted line), and after photolysis with $2.42 \times 10^{18}$ photons $\mathrm{cm}^{-2}$ (dose $=41 \mathrm{eV} / 16 \mathrm{u}$; solid line). HNCO, $\mathrm{N}_{2} \mathrm{O}, \mathrm{OCN}^{-}, \mathrm{HCN}$, and $\mathrm{CH}_{2} \mathrm{~N}_{2}$ are not detected after UV irradiation.

energy released by ions is independent of the optical constants of the sample, and at high doses impinging ions continue to release energy through the whole thickness of the sample and thus an additional modification of the sample occurs; on the other hand, the absorption coefficient of the refractory residue is higher than that of the pristine ice, and UV photons cause minor additional modifications to the deeper layers of the sample as photolysis proceeds.

The experimental data were fitted by the exponential curve

$y=\mathrm{e}^{-\sigma D}$,

where $y$ is the normalized column density of methanol, $\sigma$ is the cross section (in $16 \mathrm{u} / \mathrm{eV}$ ), and $D$ is the dose in $(\mathrm{eV} / 16 \mathrm{u})$. We obtained an average destruction cross-section for methanol equal to $0.19 \pm 0.0316 \mathrm{u} / \mathrm{eV}$.

After ion bombardment, newly formed species such as $\mathrm{CO}_{2}\left(2343 \mathrm{~cm}^{-1}\right), \mathrm{CO}\left(2138 \mathrm{~cm}^{-1}\right), \mathrm{H}_{2} \mathrm{CO}\left(1728 \mathrm{~cm}^{-1}\right), \mathrm{CH}_{4}$ $\left(1305 \mathrm{~cm}^{-1}\right), \mathrm{N}_{2} \mathrm{O}\left(2235 \mathrm{~cm}^{-1}\right), \mathrm{HNCO}\left(2263 \mathrm{~cm}^{-1}\right)$, and $\mathrm{OCN}^{-}$ $\left(2168 \mathrm{~cm}^{-1}\right)$ were detected in the ice mixture (Figs. 2 and 4$)$. There is also a broad feature at about $2080 \mathrm{~cm}^{-1}$ that may be attributed to some combination of $\mathrm{HCN}$ and $\mathrm{CH}_{2} \mathrm{~N}_{2}$ (Grim \& Greenberg 1987; Sandford \& Allamandola 1990; Elsila et al. 1997; Demyk et al. 1998; Novozamsky et al. 2001; Hudson et al. 2001; Moore \& Hudson 2003). The formation of N-bearing species indicates that the $\mathrm{N} \equiv \mathrm{N}$ bond has been broken by ions. A weak feature is present at about $2327 \mathrm{~cm}^{-1}$, which is assigned to $\mathrm{N}_{2}$. In fact, $\mathrm{N}_{2}$ has no infrared active bands because it is a homonuclear molecule; but in the solid phase the symmetry is broken and a weak band appears because of the interaction with neighboring molecules (Sandford et al. 2001).

Although methanol is destroyed for the UV photolysis, only species such as $\mathrm{CO}_{2}, \mathrm{CO}, \mathrm{H}_{2} \mathrm{CO}$, and $\mathrm{CH}_{4}$ are detected while the amount of any N-bearing species is below the detection limit (Figs. 2 and 4). As expected from the work of Hudson \& Moore (2002), $N_{3}$ was not detected. Wu et al. (2012) have shown that for very thick layers of $\mathrm{N}_{2}$ ice $(160 \mu \mathrm{m}), \mathrm{N}_{3}$ can be detected after UV photolysis. Given that the ratio of $\mathrm{N}_{3}$ to $\mathrm{N}_{2}$ formed by UV photolysis is approximately $10^{-6}$ (Wu et al. 2012), for our sample thickness of $250 \mathrm{~nm}$, where $\mathrm{N}_{2}$ constitutes about half of the ice, we predict that any $\mathrm{N}_{3}$ band at $1657 \mathrm{~cm}^{-1}$, assuming that the width is $3 \mathrm{~cm}^{-1}$, will have a peak optical depth of about

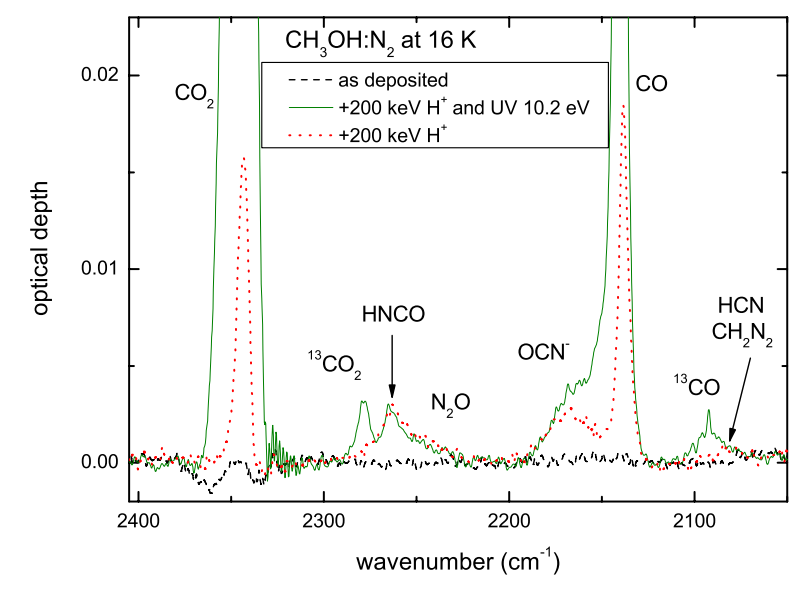

Fig. 5. IR transmittance spectra in optical depth-scale (P polarization) of $\mathrm{CH}_{3} \mathrm{OH}: \mathrm{N}_{2}$ ice (about $250 \mathrm{~nm}$ thick) deposited at $16 \mathrm{~K}$ before (dashed line), after bombardment with $1.58 \times 10^{14} 200 \mathrm{keV} \mathrm{H}^{+} \mathrm{cm}^{-2}$ alone (dose $=4 \mathrm{eV} / 16 \mathrm{u}$; dotted line) and after simultaneous processing with $1.29 \times 10^{14} 200 \mathrm{keV} \mathrm{H}^{+} \mathrm{cm}^{-2}$ and $1.76 \times 10^{18}$ photons $\mathrm{cm}^{-2}$ (total dose $=$ $37 \mathrm{eV} / 16 \mathrm{u}$; solid line). N-bearing species such as $\mathrm{HNCO}, \mathrm{N}_{2} \mathrm{O}, \mathrm{OCN}^{-}$, $\mathrm{HCN}$, and $\mathrm{CH}_{2} \mathrm{~N}_{2}$ are formed.

$5 \times 10^{-6}$, which is well below the level of noise in our spectra $\left(\tau_{\text {noise }} \sim 10^{-4}\right)$.

In comparing panels (b) and (c) in Fig. 2, it is apparent that relatively more $\mathrm{CO}$ and $\mathrm{CO}_{2}$ is produced by $\mathrm{UV}$ photolysis than by ion bombardment. As discussed in Sect. 2, the amount of CO and $\mathrm{CO}_{2}$ detected by IR spectroscopy is affected by the problem that $\mathrm{UV}$ photons cause desorption of $\mathrm{H}_{2} \mathrm{O}$ and $\mathrm{CO}_{2}$ from the walls of the vacuum chamber, followed by deposition of these species on top of the Ar layer of the sample. $\mathrm{CO}$ is then formed by UV photolysis of $\mathrm{CO}_{2}$ molecules. This effect is negligible for the ion bombardment experiments because the ion beam is well focused on the sample and does not hit the walls of the vacuum chamber.

An IR spectrum taken after the simultaneous processing of $\mathrm{CH}_{3} \mathrm{OH}: \mathrm{N}_{2}$ ice by both $\mathrm{H}^{+}$ions and $\mathrm{UV}$ photons is shown in Figs. 2 and 5. The simultaneous processes destroy methanol and cause the formation of species such as $\mathrm{CO}_{2}, \mathrm{CO}, \mathrm{H}_{2} \mathrm{CO}, \mathrm{CH}_{4}$, $\mathrm{N}_{2} \mathrm{O}, \mathrm{HNCO}, \mathrm{OCN}^{-}$, and $\mathrm{CH}_{2} \mathrm{~N}_{2}$. As expected, ion bombardment efficiently breaks up the $\mathrm{N}_{2}$ molecule to produce $\mathrm{N}$-bearing species. Also, comparatively large abundances of $\mathrm{CO}$ and $\mathrm{CO}_{2}$ are produced due to UV photolysis.

As discussed above, N-bearing species are not expected to be detected after UV photolysis in our experimental conditions. Therefore a comparison was made between the spectra of samples processed by ions and UV, and spectra relative to a sample bombarded only by ions at the same ion fluence to study the formation of N-bearing species (namely $\mathrm{HNCO}$ and $\mathrm{OCN}^{-}$) after simultaneous processing. This spectral region also contains strong ${ }^{13} \mathrm{CO}_{2}\left(2278 \mathrm{~cm}^{-1}\right)$ and $\mathrm{CO}\left(2138 \mathrm{~cm}^{-1}\right)$ bands that overlap with the HNCO $\left(2263 \mathrm{~cm}^{-1}\right)$ and $\mathrm{OCN}^{-}\left(2168 \mathrm{~cm}^{-1}\right)$ bands, respectively. To better compare the effects of the different processing, the spectrum relative to the UV photolyzed sample was subtracted from the spectrum of the ion- and UV processed sample. This corrected spectrum for simultaneous ion bombardment and UV irradiation was compared with the spectrum for the ionsonly experiment at the same ion fluence, as shown in Fig. 6. Clearly, the spectra in Fig. 6 exhibit very similar band profiles and intensities, indicating that the abundances of $\mathrm{HNCO}$ and $\mathrm{OCN}^{-}$are not affected by UV photolysis when simultaneous energetic processing takes place. 


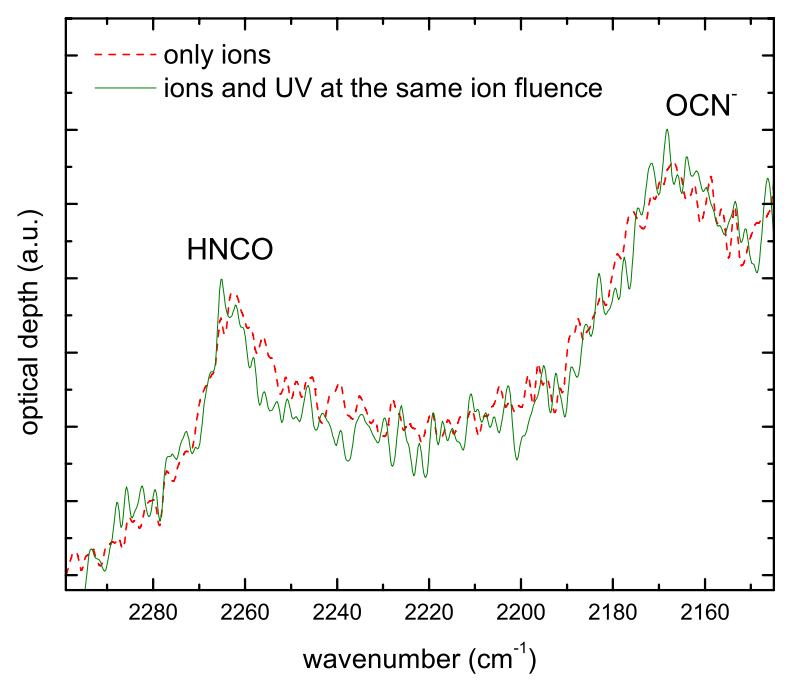

Fig. 6. IR transmittance spectra in optical-depth scale (P polarization) of $\mathrm{CH}_{3} \mathrm{OH}: \mathrm{N}_{2}$ ice (about $250 \mathrm{~nm}$ thick) deposited at $16 \mathrm{~K}$ after bombardment with $1.58 \times 10^{14} 200 \mathrm{keV} \mathrm{H}^{+} \mathrm{cm}^{-2}$ (dose $=4 \mathrm{eV} / 16 \mathrm{u}$; dashed line) and after processing by a combination of $1.29 \times 10^{14} 200 \mathrm{keV} \mathrm{H}^{+} \mathrm{cm}^{-2}$ and $1.76 \times 10^{18}$ photons $\mathrm{cm}^{-2}$ (total dose $=37 \mathrm{eV} / 16 \mathrm{u}$ ) with the band profile contribution from $\mathrm{CO}$ and ${ }^{13} \mathrm{CO}_{2}$ produced by $\mathrm{UV}$ photolysis removed (solid line). The band profiles and intensities of $\mathrm{HNCO}$ and $\mathrm{OCN}^{-}$are similar for both spectra.

Figure 7 shows the column density of $\mathrm{CH}_{4}$ as a function of dose. The column density of $\mathrm{CH}_{4}$ was derived from the spectra in optical-depth scale from the band at about $1310 \mathrm{~cm}^{-1}$ using the band strength value $A=6.4 \times 10^{-18} \mathrm{~cm} \mathrm{molecule}^{-1}$ (Mulas et al. 1998). For each experiment the $\mathrm{CH}_{4}$ column density was normalized to the initial $\mathrm{CH}_{3} \mathrm{OH}$ column density. At the beginning of processing, the $\mathrm{CH}_{4}$ column density increased rapidly; it reached the highest value when the dose increased and after further processing it started to decrease. The figure shows that at the beginning (i.e., at low dose) the formation rate of $\mathrm{CH}_{4}$ is the same regardless of the processing. On the other hand, the highest $\mathrm{CH}_{4}$ column density value reached after UV photolysis was about three times higher than the maximum amount reached after ion bombardment. As discussed above, for $\mathrm{CH}_{3} \mathrm{OH}$ destruction, this is caused by the change of the optical constants of the sample after processing. Furthermore, similarly to the discussion by Loeffler et al. (2005), in the case of ${ }^{13} \mathrm{CO}_{2}$ formation after processing of pure ${ }^{13} \mathrm{CO}$, this could be caused by the different ratio between the formation and destruction cross-section of $\mathrm{CH}_{4}$ by $200 \mathrm{keV}$ protons and Lyman-alpha photons. For the simultaneous processing, the highest $\mathrm{CH}_{4}$ column density depends on the ratio between the UV and ion dose during the experiments. When the UV dose prevails (average dose ratio $=15$ ), the highest $\mathrm{CH}_{4}$ column density is similar to the value reached after UV photolysis whereas when UV and ion doses are similar (dose ratio $=1.5$ ), the highest $\mathrm{CH}_{4}$ column density is an intermediate value between the value obtained after UV photolysis and the value obtained after ion bombardment.

The experimental data (from dose $=0$ to the dose value at which the highest $\mathrm{CH}_{4}$ column density is reached) were fitted with an exponential curve

$y=y_{\infty}\left(1-\mathrm{e}^{-\sigma D}\right)$,

where $y_{\infty}$ is the asymptotic value, $\sigma$ is the cross section (in $16 \mathrm{u} / \mathrm{eV})$ and $D$ is the dose (in $\mathrm{eV} / 16 \mathrm{u})$. The formation rate $(R$ in $16 \mathrm{u} / \mathrm{eV}$ ) was obtained from the equation

$R=y_{\infty} \times \sigma$.

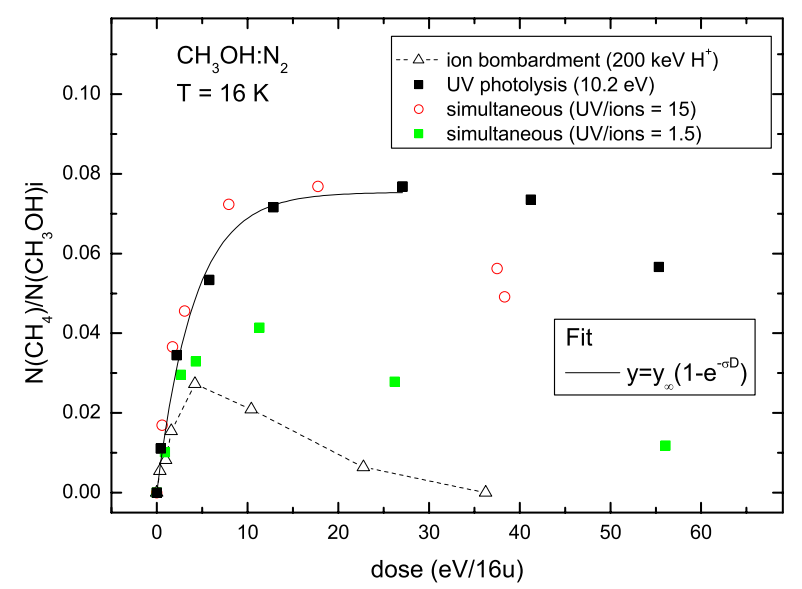

Fig. 7. Column density of $\mathrm{CH}_{4}$ formed after processing of $\mathrm{CH}_{3} \mathrm{OH}: \mathrm{N}_{2}$ mixtures at $16 \mathrm{~K}$. The $\mathrm{CH}_{4}$ column density is normalized to the initial $\mathrm{CH}_{3} \mathrm{OH}$ column density for each sample. The solid line is the fit of the experimental data relative to UV photolysis by an exponential curve. The dashed line connecting data points relative to ion bombardment has been drawn to guide the eye.

The parameters resulting from the fit and $R$ values are listed in Table 2.

\section{Discussion}

Interstellar ices are known to be simultaneously processed by both cosmic-ray bombardment and UV photolysis. It has been estimated that in dense molecular clouds the flux of cosmic ions is $1 \mathrm{~cm}^{-2} \mathrm{~s}^{-1}$ in the approximation of effective monoenergetic $1-\mathrm{MeV}$ protons (Mennella et al. 2003). This flux must be regarded as an effective quantity. It represents the flux of $1 \mathrm{MeV}$ protons, which gives rise to the ionization rate produced by the cosmic-ray spectrum if hydrogen were the only source for ionization. As discussed by Prasad \& Tarafdar (1983), an internal UV flux, $F(U V)$, is present in dense molecular clouds due to the cosmic-ray-induced fluorescence of molecular hydrogen. Depending on the assumed cosmic-ray spectrum at low energy, Shen et al. (2004) obtained $F(U V)=2 \times 10^{3}-3 \times 10^{4}$ photons $\mathrm{cm}^{-2} \mathrm{~s}^{-1}$.

In our laboratory investigations we used $200 \mathrm{keV}$ protons; based on experimental results, the best assumption we can make is that the chemical effects depend on the total dose $\left(\mathrm{eV}\right.$ molecule $\left.{ }^{-1}\right)$ deposited onto the sample, therefore these effects scale with the stopping power of impinging ions (e.g., Palumbo 2006; Sicilia et al. 2012).

Recently, Shen et al. (2004) estimated the energy deposition onto water-ice grain mantles by cosmic rays and by UV photons in dense molecular clouds. They found that depending on the assumed cosmic-ray spectrum at low energy, after $10^{7}$ years the dose deposited by UV photons varies in the range $100-10 \mathrm{eV} /$ molecule and the dose deposited by cosmic rays varies in the range $10-1 \mathrm{eV} /$ molecule. Based on these results, the energy input by UV photons is about an order of magnitude higher than the energy input by cosmic-ray particles. This means that our experimental results are fully representative of the dose range deposited by UV photons and cosmic rays on icy grain mantles in dense molecular clouds.

Moore (1999) showed that in diffuse regions UV photons deposit more energy in the icy mantles than $1 \mathrm{MeV}$ protons. In particular, after $10^{7}$ years the dose deposited by UV photons is on the order of $10^{6} \mathrm{eV} /$ molecule and the dose deposited 
F. Islam et al.: Simultaneous UV- and ion processing of ice mixtures

Table 2. Best-fit parameters for the formation of $\mathrm{CH}_{4}$ after energetic processing of a $\mathrm{CH}_{3} \mathrm{OH}: \mathrm{N}_{2}$ mixture at $16 \mathrm{~K}$.

\begin{tabular}{lccc}
\hline \hline Processing & $\begin{array}{c}\text { Asymptotic value } \\
N\left(\mathrm{CH}_{4}\right) / N\left(\mathrm{CH}_{3} \mathrm{OH}\right) \mathrm{i}\end{array}$ & $\begin{array}{c}\sigma^{a} \\
(16 \mathrm{u} / \mathrm{eV})\end{array}$ & $\begin{array}{c}R^{b} \\
(16 \mathrm{u} / \mathrm{eV})\end{array}$ \\
\hline Ion bombardment & $0.037 \pm 0.007$ & $0.3 \pm 0.1$ & $0.012 \pm 0.007$ \\
UV photolysis & $0.075 \pm 0.003$ & $0.24 \pm 0.03$ & $0.018 \pm 0.003$ \\
Simultaneous (dose ratio $=15)$ & $0.077 \pm 0.003$ & $0.34 \pm 0.04$ & $0.026 \pm 0.005$ \\
Simultaneous (dose ratio $=1.5)$ & $0.042 \pm 0.003$ & $0.39 \pm 0.06$ & $0.016 \pm 0.004$ \\
\hline
\end{tabular}

Notes. ${ }^{(a)}$ Cross section $\sigma$ given by Eq. (2). ${ }^{(b)}$ Formation rate $R$ given by Eq. (3).

by low-energy cosmic rays is on the order of $30 \mathrm{eV} /$ molecule. This result is obtained assuming that the flux of $1 \mathrm{MeV}$ protons is about $10 \mathrm{~cm}^{-2} \mathrm{~s}^{-1}$ (Strazzulla \& Johnson 1991; Indriolo \& McCall 2012) and the flux of UV photons is about $9.6 \times 10^{7}$ photons $\mathrm{cm}^{-2} \mathrm{~s}^{-1}$ (Jenniskens et al. 1993). As suggested by Westley et al. (1995a), in diffuse regions ice mantles are not observed because the photon induced desorption rate is higher than the gas adsorption rate, implying rapid removal of a pre-existing ice mantle.

In a recent work, Pedersen \& Gómez De Castro (2011) have modeled the disk of a young stellar object assuming that the $\mathrm{UV}$ radiation field of a T Tauri star at $500 \mathrm{AU}$ from the central object is $2.9 \times 10^{10}$ photons $\mathrm{cm}^{-2} \mathrm{~s}^{-1}$. Strazzulla et al. (1983b) have estimated that the flux of $1 \mathrm{MeV}$ protons in a T Tauri star is $2 \times 10^{10} \mathrm{~cm}^{-2} \mathrm{~s}^{-1}$ at $1 \mathrm{AU}$. Assuming that the flux decreases with distance as $\mathrm{d}^{-2}$, at $500 \mathrm{AU}$ the value is $8 \times 10^{4} \mathrm{~cm}^{-2} \mathrm{~s}^{-1}$. Using the same hypothesis as Moore (1999), UV photons deposit more energy in the icy mantles than $1 \mathrm{MeV}$ protons also in circumstellar regions. However, when planetesimals form, UV photons are absorbed in the external layers (on the order of $10^{2} \mathrm{~nm}$ ) while energetic ions can penetrate the bodies and release their energy in deeper layers.

As another example, it has been shown that cosmic rays can alter the outer few meters of a cometary nucleus. On the other hand, the penetration depth of UV photons is lower than that of ions and their contribution can be neglected (e.g., Strazzulla \& Johnson 1991; Modica et al. 2012). It has been estimated that near the surface the dose deposited by cosmic rays after $10^{9}$ years is as high as hundreds of $\mathrm{eV} / 16 \mathrm{u}$; therefore, volatile species eventually formed by cosmic-ray bombardment would be quickly destroyed by the same mechanism. At deeper layers from the surface, the absorbed dose decreases and doses as low as a few eV/16u, still able to produce noticeable chemical effects, are accumulated at depths on the order of tens of meters.

We have considered a $\mathrm{CH}_{3} \mathrm{OH}: \mathrm{N}_{2}$ mixture. It might be argued that observations indicate that water ice is the most abundant solid-phase species in cometary and interstellar ices. Accordingly, the study of water-rich ice mixtures should be considered. However, in our experiments the initial thickness of the ice samples was chosen as a compromise between a sample thick enough to obtain infrared spectra with a good signal-to-noise ratio and thin enough to be uniformly processed by ions and UV photons. If we add water to the mixture (without increasing the thickness of the sample), we reduce the amount of $\mathrm{CH}_{3} \mathrm{OH}$ and $\mathrm{N}_{2}$, reducing in turn the amount of $\mathrm{N}$-bearing species formed after processing. This reduction would make the detection of these species even more difficult. On the other hand, recent laboratory experiments (e.g., Fuchs et al. 2009) have shown that $\mathrm{CH}_{3} \mathrm{OH}$ molecules efficiently form after hydrogenation of $\mathrm{CO}$ molecules in CO-rich and water-poor ices. Therefore it is reasonable to assume that $\mathrm{CH}_{3} \mathrm{OH}$-rich and water-poor ice mantles may exist along the line of sight of dense molecular clouds (e.g., Skinner et al. 1992; Palumbo \& Strazzulla 1992; Cuppen et al. 2011).

Most of our knowledge on the effects of UV photons and low-energy ions on astrophysically relevant ices is based on laboratory simulations. In the past 35 years, several experiments have been performed in different laboratories to study these effects separately but, to the best of our knowledge, never simultaneously. Here we have presented an experimental study of the effects of simultaneous processing on a $\mathrm{CH}_{3} \mathrm{OH}: \mathrm{N}_{2}$ mixture at $16 \mathrm{~K}$. Our results confirm that when UV photolysis and ion bombardment act separately, from a qualitative point of view their effects are very similar but significant quantitative differences may exist. We did not detect any synergistic effect after simultaneous processing, but from a quantitative point of view the behavior of newly formed molecules (such as $\mathrm{CH}_{4}$, see Fig. 7) can in some instances significantly depend on the UV/ions dose ratio. Hence the effects of simultaneous processing on other astrophysically relevant mixtures should be experimentally investigated to better understand the chemical evolution of solid phase molecules in space.

Acknowledgements. This research has been financially supported by the Italian Ministero dell'Istruzione, dell'Università e della Ricerca (PRINMIUR) and by the LASSIE Initial Training Network, which is supported by the European Community's Seventh Framework Programme under Grant Agreement Number 238258.

\section{Appendix A: Monitoring system of the UV flux during irradiation}

We developed a monitoring system of the UV flux to obtain a reliable measure of the total number of UV photons that reach the sample during photolysis (see Baratta et al. 2002). This was obtained by measuring the current produced by the photoelectric effect on a platinum wire placed at the end of the aluminium light collector. The integrated current $C(\mu \mathrm{C})$ measured by the wire detector is proportional to the photon fluence $\Phi_{\mathrm{ph}}\left(\mathrm{cm}^{-2}\right)$, which is the total UV photon number per square centimeter that reaches the sample. In particular,

$\Phi_{\mathrm{ph}}=M_{\mathrm{cal}} \cdot C$

where $M_{\text {cal }}$ is the calibration constant. The calibration constant of the wire was determined by measuring the $\mathrm{O}_{2}$ to $\mathrm{O}_{3}$ conversion rate in a UV photolyzed $\mathrm{O}_{2}$ ice film (see Gerakines et al. 1996, 2000; Baratta et al. 2002). Following Gerakines et al. (2000), we assumed a conversion efficiency of $\mathrm{O}_{2}$ to $\mathrm{O}_{3}$ equal to the quantum yield $Y$ measured for the gas phase by Groth (1937), corresponding to 1.92 ozone molecules produced for each absorbed UV photon in the $\mathrm{O}_{2}$ film. The ozone production was monitored through the $1040 \mathrm{~cm}^{-1}$ band by using a band strength value of $1.4 \times 10^{-17} \mathrm{~cm}$ molecule ${ }^{-1}$ (Smith et al. 1985). If we consider an $\mathrm{O}_{2}$ film of thickness $t(\mathrm{~cm})$, the column density 


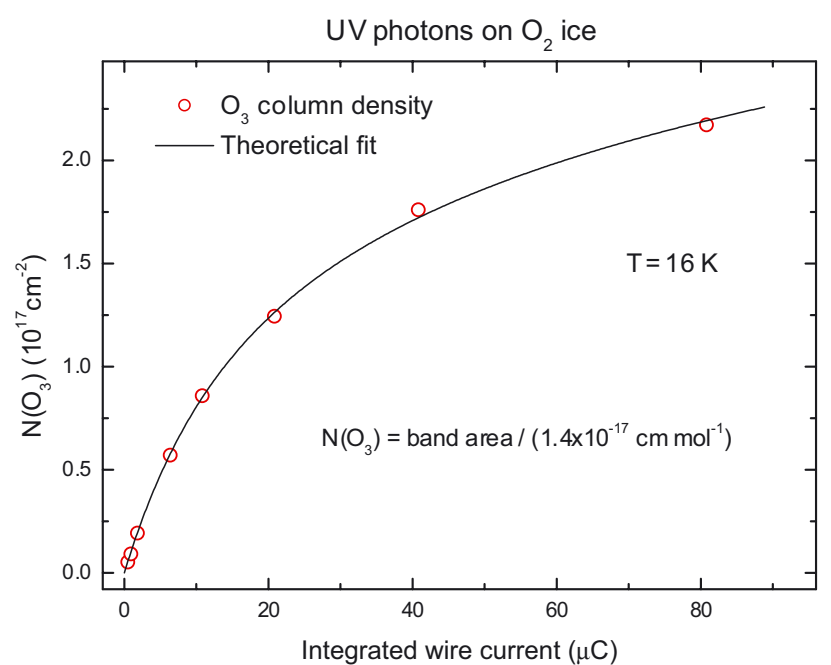

Fig. A.1. $\mathrm{O}_{3}$ column density at different photon fluences as a function of integrated current detected. A theoretical fit obtained by a nonlinear least-squares routine is used to provide $n^{\infty}, \alpha$, and $s$ as best-fit parameters.

of $\mathrm{O}_{3}$ molecules $N\left(\Phi_{\mathrm{ph}}, t\right)\left(\mathrm{cm}^{-2}\right)$ formed in the film after a photon fluence $\Phi_{\mathrm{ph}}\left(\mathrm{cm}^{-2}\right)$ can be computed through the equation

$N\left(\Phi_{\mathrm{ph}}, t\right)=n^{\infty} \int_{0}^{t}\left(1-\mathrm{e}^{-\sigma \Phi \mathrm{e}^{-\alpha x}}\right) \mathrm{d} x$

where $n^{\infty}$ is the $\mathrm{O}_{3}$ number density at saturation $\left(\mathrm{cm}^{-3}\right)$, that is, when the number of $\mathrm{O}_{3}$ molecules formed $\left(\mathrm{O}_{2}\right.$ to $\left.\mathrm{O}_{3}\right)$ is equal to the number of $\mathrm{O}_{3}$ molecules destroyed $\left(\mathrm{O}_{3}\right.$ to $\left.\mathrm{O}_{2}\right) ; \alpha$ is the absorption coefficient $\left(\mathrm{cm}^{-1}\right)$ at Lyman-alpha of the irradiated $\mathrm{O}_{2}$ film and $\sigma$ is the cross section of the process $\left(\mathrm{cm}^{2}\right)$. Equation (A.2) takes into account the exponential decay of the UV flux with the depth $x(\mathrm{~cm})$ within the film. By substituting Eq. (A.1) in Eq. (A.2), the column density can be written versus the integrated current,

$N(C, t)=n^{\infty} \int_{0}^{t}\left(1-\mathrm{e}^{-s C \mathrm{e}^{-\alpha x}}\right) \mathrm{d} x$,

where $s=\sigma \cdot M_{\text {cal }}$. Equation (A.3) can be used to fit the experimental data acquired during the calibration experiment. In Fig. A.1, the $\mathrm{O}_{3}$ column density at different photon fluences versus the integrated current is reported together with the theoretical fit obtained by a nonlinear least-squares routine that provides $n^{\infty}$, $\alpha$, and $s$ as best-fit parameters. The film thickness $t$ in Eq. (A.3) was computed by using the laser interference method.

The quantum yield $Y$ can be related to the initial $\mathrm{O}_{3}$ formation rate when $t$ approaches $\infty$ in Eq. (A.3). Indeed, in this case, all the UV photons are absorbed by an oxygen molecule since the amount of $\mathrm{O}_{3}$ molecules is negligible at very low fluence.

It follows that

$\lim _{C \rightarrow 0} \frac{\mathrm{d} N(C, \infty)}{\mathrm{d} C}=Y \frac{\mathrm{d} \Phi_{\mathrm{ph}}}{\mathrm{d} C}$.

From Eq. (A.3):

$\lim _{C \rightarrow 0} \frac{\mathrm{d} N(C, \infty)}{\mathrm{d} C}=\frac{n^{\infty} \cdot s}{\alpha}$.

From Eq. (A.1):

$\frac{\mathrm{d} \Phi_{\mathrm{ph}}}{\mathrm{d} C}=M_{\mathrm{cal}}$, and finally,

$M_{\text {cal }}=\frac{n^{\infty} \cdot s \cdot \beta}{\alpha \cdot Y}$,

where the $\beta$ factor $(=0.93)$ takes into account a $10 \%$ UV photon loss by reflection and diffusion and the increased optical path due to the IR beam oblique incidence. The calibration constant depends on the geometry of the experimental set-up, that is, the relative positions of the microwave cavity of the lamp, the aluminium light collector, and the target. The geometry can be slightly different each time the UV lamp is mounted on the vacuum chamber; for this reason we performed the calibration every time the UV lamp was mounted. We found that the calibration constant was roughly the same before and after each UV photolysis section, and it varied in the range between $4 \times 10^{15}$ and $6 \times 10^{15} \mathrm{~cm}^{-2} \mu \mathrm{C}^{-1}$ among different sections. The flux of the lamp can be adjusted in the range $10^{12}-5 \times 10^{14} \mathrm{~cm}^{-2} \mathrm{~s}^{-1}$.

\section{References}

Allamandola, L. J., Sandford, S. A., \& Valero, G. J. 1988, Icarus, 76, 225 Baratta, G. A., \& Palumbo, M. E. 1998, J. Opt. Soc. Am. A, 15, 3076

Baratta, G. A., Spinella, F., Leto, G., Strazzulla, G., \& Foti, G. 1991, A\&A, 252, 421

Baratta, G. A., Palumbo, M. E., \& Strazzulla, G. 2000, A\&A, 357, 1045 Baratta, G. A., Leto, G., \& Palumbo, M. E. 2002, A\&A, 384, 343

Brown, W. L., Lanzerotti, L. J., \& Johnson, R. E. 1982, Science, 218, 525

Cottin, H., Szopa, C., \& Moore, M. H. 2001, ApJ, 561, L139

Cuppen, H. M., Penteado, E. M., Isokoski, K., van der Marel, N., \& Linnartz, H. 2011, MNRAS, 417, 2809

Demyk, K., Dartois, E., d'Hendecourt, L., et al. 1998, A\&A, 339, 553

Elsila, J., Allamandola, L. J., \& Sandford, S. A. 1997, ApJ, 479, 818

Ferini, G., Baratta, G. A., \& Palumbo, M. E. 2004, A\&A, 414, 757

Fuchs, G., W., Cuppen, H. M., Ioppolo, S., et al. 2009, A\&A, 505, 629

Fulvio, D., Sivaraman, B., Baratta, G. A., Palumbo, M. E., \& Mason, N. J. 2009, Spectrochimica Acta Part A: Molecular Spectroscopy, 72, 1007

Garozzo, M., La Rosa, L., Kanuchova, Z., et al. 2011, A\&A, 528, A118

Gerakines, P. A., \& Moore, M. H. 2001, Icarus, 154, 372

Gerakines, P. A., Schutte, W. A., \& Ehrenfreund, P. 1996, A\&A, 312, 289

Gerakines, P. A., Moore, M. H., \& Hudson, R. L. 2000, A\&A, 357, 793

Grim, R. J. A., \& Greenberg, J. M. 1987, ApJ, 321, L91

Grim, R. J. A., Greenberg, J. M., de Groot, M. S., et al. 1989, A\&AS, 78, 161

Groth, W. 1937, Z. Phys. Chem., 37, 307

Hagen, W., Allamandola, L. J., \& Greenberg, J. M. 1979, Ap\&SS, 65, 215

Hudson, R. L., \& Moore, M. H. 2002, ApJ, 568, 1095

Hudson, R. L., Moore, M. H., \& Gerakines, P. A. 2001, ApJ, 550, 1140

Hudson, R. L., Palumbo, M. E., Strazzulla, G., et al. 2008, in The Solar System Beyond Neptune, 507

Indriolo, N., \& McCall, B. J. 2012, ApJ, 745, 91

Jamieson, C. S., \& Kaiser, R. I. 2007, Chem. Phys. Lett., 440, 98

Jenniskens, P., Baratta, G. A., Kouchi, A., et al. 1993, A\&A, 273, 583

Johnson, R. E., Grosjean, D. E., Jurac, S., \& Baragiola, R. A. 1993, EOS, 74, 572

Kobayashi, K., Ogawa, T., Tonishi, H., et al. 2008, Electron. Commun. Jap., 91, 293

Leto, G., \& Baratta, G. A. 2003, A\&A, 397, 7

Loeffler, M. J., Baratta, G. A., Palumbo, M. E., Strazzulla, G., \& Baragiola, R. A. 2005, A\&A, 435, 587

Mennella, V., Baratta, G. A, Esposito, A., Ferini, G., \& Pendleton, Y. J. 2003, ApJ, 587, 727

Modica, P., \& Palumbo, M. E. 2010, A\&A, 519, A22

Modica, P., Palumbo, M. E. \& Strazzulla, G. 2012, Planet Space Science, 73, 425

Moore, M. H. 1999, in Solid Interstellar Matter: the ISO Revolution, eds. L. d'Hendecourt, C. Joblin, \& A. Jones (New York: Springer-Verlag,), 199

Moore, M. H., \& Hudson, R. L. 2003, Icarus, 161, 486

Moore, M. H., Donn, B., Khanna, R., \& A'Hearn, M. F. 1983, Icarus, 54, 388

Moore, M. H., Hudson, R. L., \& Gerakines, P. A. 2001, Spectrochimica Acta, 57, 843

Mulas, G., Baratta, G. A., Palumbo, M. E., \& Strazzulla, G. 1998, A\&A, 333, 1025

Novozamsky, J. H., Schutte, W. A., \& Keane J. V. 2001, A\&A, 379, 588 
F. Islam et al.: Simultaneous UV- and ion processing of ice mixtures

Öberg, K. I., Garrod, R. T., van Dishoeck, E. F., \& Linnartz, H. 2009, A\&A, 504, 891

Palumbo, M. E. 2006, A\&A 453, 903

Palumbo, M. E., \& Strazzulla, G. 1992, A\&A, 259, L12

Palumbo, M. E., Baratta, G. A., Brucato, J. R., et al. 1998, A\&A, 334, 247

Palumbo, M. E., Castorina, A. C., \& Strazzulla, G. 1999, A\&A, 342, 551

Palumbo, M. E., Ferini, G., \& Baratta, G. A. 2004, Adv. Space Res., 33, 49

Palumbo, M. E., Baratta, G. A., Leto, G., \& Strazzulla, G. 2010, J. Mol. Structure, 972, 64

Pedersen, A., \& Gómez De Castro, A. I. 2011, ApJ, 740, 77

Prasad, S. S., \& Tarafdar, S. P. 1983, ApJ, 267, 603

Roux, J. A., Wood, B. E., Smith, A. M., \& Plyler, R. R. 1980, Arnold Engineering Development Center Final Report AEDC-TR-79-81 (Arnold Air Force Station)

Sandford, S. A., \& Allamandola, L. J. 1990, ApJ, 355, 357

Sandford, S. A., Bernstein, M. P., Allamandola, L. J., Goorvitch, D., \& Teixeira, T. C. V. S. 2001, ApJ, 548, 836

Shemansky, D. E., Matheson, P., Hall, D. T., Hu, H.-Y., \& Tripp, T. M. 1993, Nature, 363, 329

Shen, C. J., Greenberg, J. M., Schutte, W. A., \& van Dishoek, E. F. 2004, A\&A, 415,203
Sicilia, D., Ioppolo, S., Vindigni, T., Baratta, G. A., \& Palumbo, M. E. 2012, A\&A, 543, A155

Skinner, C. J., Tielens, A. G. G. M., Barlow, M. J., \& Justtanont, K. 1992, ApJ, 399, L79

Smith, M. A. H., Rinsland, C. P., Fridovich, B., \& Rao, K. N. 1985, in Molecular Spectroscopy: Modern Research, Vol. III, ed. K. N. Rao (Academic Press, London), 111

Strazzulla, G., \& Johnson, R. E. 1991, in Comets in the post-Halley era, 1 (Dordrecht: ASSL Series), 243

Strazzulla, G., Pirronello, V., \& Foti, G. 1983a, A\&A, 123, 93

Strazzulla, G., Pirronello, V., \& Foti, G. 1983b, ApJ, 271, 255

Strazzulla, G., Baratta, G. A., \& Palumbo, M. E. 2001, Spectrochim. Acta A, 57, 825

Swordy, S. P. 2001, Space Sci. Rev., 99, 85

Westley, M. S., Baragiola, R. A., Johnson, R. E., \& Baratta, G. A. 1995a, Nature, 373,405

Westley, M. S., Baragiola, R. A., Johnson, R. E., \& Baratta, G. A. 1995b, Planet Space Sci., 43, 1311

Wu, Y. J., Wu, C. Y. R., Chou, S. L., et al. 2012, ApJ, 746, 175

Ziegler, J. F., Ziegler, M. D., \& Biersack, J. P. 2008, The stopping and range of ions in solids (New York: Pergamon Press), http://www. srim.org 